\title{
On injective and divisible modules
}

Received: 30 October 2010 / Accepted: 6 March 2011 / Published online: 24 March 2012

(C) The Author(s) 2012. This article is published with open access at Springerlink.com

\begin{abstract}
Divisible modules over general rings are considered and a general notion of divisibility is defined. In order to study these divisible modules we generalize the notion of injectivity. One consequence is that rings for which every principal right ideal is projective can be characterized. In addition, a characterization is given of when a submodule of a projective module is projective.
\end{abstract}

Mathematical Subject Classification 16D50 - 16D40

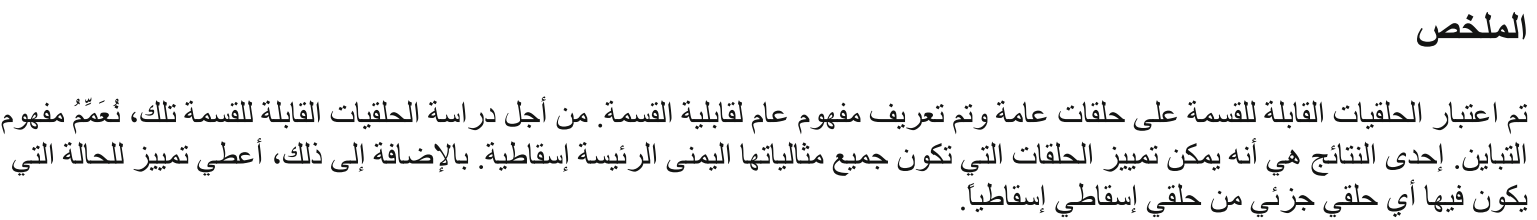

\section{Generalizations of injective modules}

All rings have an identity element and all modules are unitary right modules. Let $R$ be a ring and $M$ and $X$ be $R$-modules. Let $\mathfrak{A}$ be a non-empty collection of submodules of $M$. The module $X$ will be called $\mathfrak{A}-M$ injective in case, for every submodule $N$ in $\mathfrak{A}$, every homomorphism $\varphi: N \rightarrow X$ lifts to $M$. For a related concept see [2]. In particular, if $\mathfrak{A}$ is the collection of all submodules of $M$ then we shall call $X M$-injective, as usual (see, for example, [19, Section 16]). Note that if $\mathfrak{E}$ denotes the collection of essential submodules of $M$, then every $\mathfrak{E}-M$-injective module is $M$-injective because every submodule of $M$ is a direct summand of an essential submodule. In [13] and [15], a module $X$ is called finitely injective in case, for every $R$-module $M, X$ is $\mathfrak{F}(M)-M$-injective where $\mathfrak{F}(M)$ denotes the collection of finitely generated submodules of $M$. If $N$ is a submodule of $M$, then $N$ is $\{N\}-M$-injective if and only if $N$ is a direct summand of $M$. It follows that if $\mathfrak{S}$ denotes the collection of direct summands of $M$, then for a given collection $\mathfrak{A}$ of submodules of $M$, every $R$-module is $\mathfrak{A}-M$-injective if and only if $\mathfrak{A} \subseteq \mathfrak{S}$. For any unexplained terminology see [19].

Again let $R$ be a ring, $M$ and $X R$-modules and let $\mathfrak{A}$ be a non-empty collection of submodules of $M$. It is clear that $X$ is $\mathfrak{A}-M$-injective if and only if $X$ is $\{L\}-M$-injective for every submodule $L$ of $\mathfrak{A}$, and in this case $X$ is $\mathfrak{B}-M$-injective for every non-empty subfamily $\mathfrak{B}$ of $\mathfrak{A}$. Let $\mathfrak{A}_{M}(X)$ denote the collection of all submodules $N$ of $M$ such that $X$ is $\{N\}-M$-injective. Then $X$ is $\mathfrak{A}-M$-injective if and only if $\mathfrak{A} \subseteq \mathfrak{A}_{M}(X)$. In particular, $X$ is $\mathfrak{A}_{M}(X)-M$-injective. Note the following simple fact.

P. F. Smith $(\varangle)$

Department of Mathematics, University of Glasgow, Glasgow G12 8QW, Scotland, UK

E-mail: Patrick.Smith@glasgow.ac.uk 
Proposition 1.1 Let $R$ be any ring and let $\pi: M \rightarrow M^{\prime}$ be any epimorphism of $R$-modules. Let $\mathfrak{A}$ be any non-empty collection of submodules of $M$, each containing the kernel of $\pi$, and let $\mathfrak{A}^{\prime}$ denote the collection of submodules of $M^{\prime}$ of the form $\pi(L)$ where $L$ is any submodule of $\mathfrak{A}$. Then every $\mathfrak{A}-M$-injective $R$-module is $\mathfrak{A}^{\prime}-M^{\prime}$-injective.

Proof Let $X$ be any $\mathfrak{A}-M$-injective module. Let $L^{\prime}$ be any submodule in $\mathfrak{A}^{\prime}$ and let $\varphi: L^{\prime} \rightarrow X$ be any homomorphism. There exists a submodule $L$ in $\mathfrak{A}$ such that $L^{\prime}=\pi(L)$. Let $\pi^{\prime}: L \rightarrow L^{\prime}$ be the homomorphism defined by $\pi^{\prime}(x)=\pi(x)(x \in X)$. Then $\varphi \pi^{\prime}: L \rightarrow X$ is a homomorphism. By hypothesis, $\varphi \pi^{\prime}$ can be lifted to a homomorphism $\theta: M \rightarrow X$. Let $K$ denote the kernel of $\pi$. Note that $K \subseteq L$ and

$$
\theta(K)=\varphi \pi^{\prime}(K)=\varphi(0)=0
$$

so that $\theta$ induces a homomorphism $\theta^{\prime}: M^{\prime} \rightarrow X$ defined by $\theta^{\prime}(\pi(m))=\theta(m)$ for all $m \in M$. For each $n^{\prime} \in L^{\prime}$ there exists $n \in L$ such that $n^{\prime}=\pi^{\prime}(n)$ and hence

$$
\theta^{\prime}\left(n^{\prime}\right)=\theta(n)=\varphi \pi^{\prime}(n)=\varphi\left(n^{\prime}\right) .
$$

Thus $\theta^{\prime}$ lifts $\varphi$ to $M^{\prime}$. The result follows.

Next we consider some basic properties of $\mathfrak{A}-M$-injective modules.

Lemma 1.2 Let $R$ be a ring and let $\mathfrak{A}$ be any non-empty collection of submodules of an $R$-module $M$. Then every direct product of $\mathfrak{A}-M$-injective $R$-modules is $\mathfrak{A}-M$-injective.

Proof Clear.

Corollary 1.3 Let $R$ be a ring and let $\mathfrak{A}$ be any non-empty collection of finitely generated submodules of an $R$-module $M$. Then every direct sum of $\mathfrak{A}-M$-injective $R$-modules is $\mathfrak{A}-M$-injective.

Proof By Lemma 1.2.

Lemma 1.4 Let $R$ be a ring and let $\mathfrak{A}$ be any non-empty collection of finitely generated submodules of an $R$-module $M$. Let $Y$ be any $\mathfrak{A}-M$-injective submodule of an $R$-module $X$. Then there exists a maximal $\mathfrak{A}-M$-injective submodule $Z$ of $X$ containing $Y$.

Proof Let $\mathcal{S}$ denote the collection of all $\mathfrak{A}-M$-injective submodules of $X$ containing $Y$. Clearly $\mathcal{S}$ is nonempty. Let $U_{i}(i \in I)$ be any chain in $\mathcal{S}$ and let $U=\cup_{i \in I} U_{i}$. Then $U$ is a submodule of $X$ containing $Y$. Let $N$ be any submodule in $\mathfrak{A}$ and let $\varphi: N \rightarrow U$ be any homomorphism. Because $N$ is finitely generated, there exists $j \in I$ such that $\varphi(N) \subseteq U_{j}$ and hence $\varphi$ lifts to $M$. It follows that $U$ is $\mathfrak{A}-M$-injective. By Zorn's Lemma $\mathcal{S}$ has a maximal member.

Let $R$ be a ring and $X$ any $R$-module. Recall that an $R$-module $M$ is $X$-projective provided that for every $R$-module $X^{\prime}$, epimorphism $\pi: X \rightarrow X^{\prime}$ and homomorphism $\varphi: M \rightarrow X^{\prime}$ there exists a homomorphism $\theta: M \rightarrow X$ such that $\varphi=\pi \theta$.

Lemma 1.5 Let $R$ be any ring and let $X$ and $M$ be $R$-modules such that $M$ is $X$-projective. Let $\mathfrak{A}$ be a non-empty collection of submodules of $M$ and let $Y$ be a submodule of $X$ such that $Y$ and $X / Y$ are both $\mathfrak{A}-M$-injective. Then $X$ is $\mathfrak{A}-M$-injective.

Proof Let $N$ be any submodule in $\mathfrak{A}$ and let $\varphi: N \rightarrow X$ be any homomorphism. Let $\pi: X \rightarrow X / Y$ denote the canonical projection and $\iota: N \rightarrow M$ the inclusion mapping. Then $\pi \varphi: N \rightarrow X / Y$ is a homomorphism. By hypothesis, there exists a homomorphism $\theta: M \rightarrow X / Y$ such that $\pi \varphi=\theta \iota$. Because $M$ is $X$-projective, there exists a homomorphism $\bar{\theta}: M \rightarrow X$ such that $\theta=\pi \bar{\theta}$. Note that for all $u \in N$,

$$
\theta(u)=\varphi(u)+Y=\bar{\theta}(u)+Y,
$$

and hence $\varphi(u)-\bar{\theta}(u) \in Y$. Define a mapping $\alpha: N \rightarrow Y$ by

$$
\alpha(u)=\varphi(u)-\bar{\theta}(u)(u \in N) .
$$

Clearly $\alpha$ is a homomorphism. By hypothesis, there exists a homomorphism $\beta: M \rightarrow Y$ such that $\alpha=\beta \iota$. Let $\lambda$ denote the homomorphism $\bar{\theta}+\beta: M \rightarrow X$. For all $u \in N$,

$$
\lambda(u)=\bar{\theta}(u)+\beta(u)=\bar{\theta}(u)+\alpha(u)=\varphi(u) .
$$

It follows that $X$ is $\mathfrak{A}-M$-injective. 
Corollary 1.6 Let $R$ be any ring and let $X$ and $M$ be $R$-modules such that $M$ is $X$-projective. Let $\mathfrak{A}$ be a non-empty collection of finitely generated submodules of $M$ and let $Y$ be a submodule of $X$ such that $Y$ is $\mathfrak{A}-M$-injective. Then there exists a submodule $Z$ of $X$ containing $Y$ such that $Z$ is $\mathfrak{A}-M$-injective and $X / Z$ has no non-zero $\mathfrak{A}-M$-injective submodule.

Proof By Lemmas 1.4 and 1.5.

For any ring $R$ and $R$-module $M$, the injective hull of $M$ will be denoted by $\mathrm{E}(M)$. We now characterize when a submodule of a projective module is projective.

Theorem 1.7 Let $R$ be any ring. Then the following statements are equivalent for a submodule $M$ of a projective $R$-module $P$ :

(i) $M$ is projective.

(ii) Every homomorphic image of an $\{M\}-P$-injective module is $\{M\}-P$-injective.

(iii) Every homomorphic image of a $P$-injective module is $\{M\}-P$-injective.

(iv) Every homomorphic image of an injective $R$-module is $\{M\}-P$-injective.

Proof (i) $\Rightarrow$ (ii) Let $X$ be any $R$-module which is $\{M\}-P$-injective and let $Y$ be a submodule of $X$. Let $\alpha: M \rightarrow X / Y$ be a homomorphism. Let $\iota: M \rightarrow P$ denote the inclusion mapping and let $\pi: X \rightarrow X / Y$ denote the canonical projection mapping. Because $M$ is projective, there exists a homomorphism $\beta: M \rightarrow X$ such that $\pi \beta=\alpha$. But $X$ is $\{M\}-P$-injective, so that there exists a homomorphism $\gamma: P \rightarrow X$ with $\beta=\gamma \iota$. Note that $\pi \gamma: P \rightarrow X / Y$ is a homomorphism such that $(\pi \gamma) \iota=\pi \beta=\alpha$. It follows that $X / Y$ is $\{M\}-P$-injective.

(ii) $\Rightarrow$ (iii) $\Rightarrow$ (iv) Clear.

(iv) $\Rightarrow$ (i) Let $N$ be any $R$-module and let $L$ be a submodule of $N$. We can think of $N$ as a submodule of its injective hull $\mathrm{E}(N)$. Let $\iota_{1}: N \rightarrow \mathrm{E}(N)$ and $\iota_{2}: N / L \rightarrow \mathrm{E}(N) / L$ denote the inclusions and let $\pi_{1}: N \rightarrow N / L$ and $\pi_{2}: \mathrm{E}(N) \rightarrow \mathrm{E}(N) / L$ denote the canonical projections. Let $\theta: M \rightarrow N / L$ be any homomorphism. Since the module $\mathrm{E}(N) / L$ is $\{M\}-P$-injective it follows that there exists a homomorphism $\mu: P \rightarrow E(N) / L$ such that $\mu \iota=\iota_{2} \theta$, where again $\iota: M \rightarrow P$ denotes inclusion. But $P$ is projective, so that there exists a homomorphism $v: P \rightarrow \mathrm{E}(N)$ such that $\pi_{2} v=\mu$. Let $m \in M$. Then

$$
v(m)+L=\pi_{2} v(m)=\mu(m)=\iota_{2} \theta(m) \in N / L,
$$

and it follows that $v(m) \in N$. Thus $v(m) \in N$ for all $m \in M$. Note that $\nu \iota: M \rightarrow N$ is a homomorphism such that $\pi_{1} v \iota=\mu \iota=\theta$. It follows that the module $M$ is projective.

Corollary 1.8 Let $R$ be a ring and $\mathfrak{A}$ a non-empty collection of submodules of a projective $R$-module $P$. Then the following statements are equivalent:

(i) Every submodule in $\mathfrak{A}$ is projective.

(ii) Every homomorphic image of an $\mathfrak{A}-P$-injective module is $\mathfrak{A}-P$-injective.

(iii) Every homomorphic image of an injective $R$-module is $\mathfrak{A}-P$-injective.

Proof By Theorem 1.7.

In particular, in Corollary 1.8 in case $P=R_{R}$ we have the following immediate corollary:

Corollary 1.9 Let $R$ be a ring and $\mathfrak{A}$ a non-empty collection of right ideals of $R$. Then the following statements are equivalent:

(i) Every right ideal in $\mathfrak{A}$ is projective.

(ii) Every homomorphic image of an $\mathfrak{A}-R_{R}$-injective module is $\mathfrak{A}-R_{R}$-injective.

(iii) Every homomorphic image of an injective $R$-module is $\mathfrak{A}-R_{R}$-injective.

The equivalence (i) $\Leftrightarrow$ (iii) in Corollary 1.9, can be found in [6] in case $\mathfrak{A}$ is the collection of principal right ideals of $R$. Moreover, in case $\mathfrak{A}$ is the collection of all right ideals of $R$ then an $R$-module $X$ is injective if and only if it is $\mathfrak{A}-R_{R}$-injective by Baer's Lemma (see, for example, [19, 16.4]). Thus Corollary 1.9 generalizes the well-known fact that a ring is right hereditary if and only if every homomorphic image of an injective right $R$-module is injective (see, for example, $[19,39.16]$ ). 
Let $R$ be a ring and let $M$ be a right $R$-module. For any non-empty subset $T$ of $R, a n n_{M}(T)$ will denote the set of elements $m \in M$ such that $m t=0$ for all $t \in T$. Note that $a n n_{M}(T)$ is a subgroup of the Abelian group $(M,+)$. We now consider right $R$-modules $X$ such that $X$ is $\{A\}-R_{R}$-injective for some right ideal $A$ of $R$. Note that this occurs precisely when

$$
\operatorname{Ext}_{R}^{1}(R / A, X)=0
$$

To see why this is the case, note that the exact sequence

$$
0 \rightarrow A \rightarrow R \rightarrow R / A \rightarrow 0
$$

induces the exact sequence

$$
\operatorname{Hom}(R, X) \rightarrow \operatorname{Hom}(A, X) \rightarrow \operatorname{Ext}^{1}(R / A, X) \rightarrow \operatorname{Ext}^{1}(R, X)=0 .
$$

Thus $\operatorname{Ext}^{1}(R / A, X)=0$ if and only if the homomorphism $\operatorname{Hom}(R, X) \rightarrow \operatorname{Hom}(A, X)$ is an epimorphism.

Theorem 1.10 Let $A$ and $B$ be right ideals of a ring $R$ and let $X$ be an $R$-module such that $X$ is both $\{A\}-R_{R}$-injective and $\{B\}-R_{R}$-injective. Then $X$ is $\{A+B\}-R_{R}$-injective if and only if ann $X(A \cap B)=$ $a n n_{X}(A)+a n n_{X}(B)$.

Proof Suppose first that $a n n_{X}(A \cap B)=a n n_{X}(A)+a n n_{X}(B)$. Let $\varphi: A+B \rightarrow X$ be any homomorphism. Then $\left.\varphi\right|_{A}: A \rightarrow X$ lifts to $R$ and hence there exists an element $x$ in $X$ such that $\varphi(a)=x a$ for all $a \in A$. Similarly, there exists an element $y \in X$ such that $\varphi(b)=y b(b \in B)$. Let $c \in A \cap B$. Note that $\varphi(c)=x c=y c$ so that $(x-y) c=0$. It follows that $x-y \in a n n_{X}(A \cap B)$. By hypothesis, there exist elements $u \in a n n_{X}(A)$ and $v \in \operatorname{ann}_{X}(B)$ such that $x-y=u+v$. Let $z=x-u=y+v \in X$. For each $a \in A$,

$$
\varphi(a)=x a=(x-u) a=z a .
$$

Similarly,

$$
\varphi(b)=y b=(y+v) b=z b(b \in B) .
$$

It follows that $\varphi(a+b)=z(a+b)$ for all $a \in A, b \in B$. Thus $\varphi$ lifts to $R$. Hence $X$ is $\{A+B\}-R_{R}$-injective.

Conversely, suppose that $X$ is $\{A+B\}-R_{R}$-injective. Clearly $a n n_{X}(A) \subseteq a n n_{X}(A \cap B)$ and $a n n_{X}(B) \subseteq$ $a n n_{X}(A \cap B)$ so that $a n n_{X}(A)+a n n_{X}(B) \subseteq a n n_{X}(A \cap B)$. Now let $w \in a n n_{X}(A \cap B)$. Define a mapping $\alpha: A+B \rightarrow X$ by

$$
\alpha(a+b)=w a(a \in A, b \in B) .
$$

Note that if $a \in A$ and $b \in B$ such that $a+b=0$ then $a=-b \in A \cap B$ so that $w a=0$. It follows that the mapping $\alpha$ is well defined. Clearly $\alpha$ is a homomorphism. By hypothesis there exists an element $w^{\prime} \in X$ such that $\alpha(a+b)=w^{\prime}(a+b)$ for all $a \in A, b \in B$. Now note that

$$
w a=\alpha(a)=w^{\prime} a(a \in A),
$$

so that $w-w^{\prime} \in \operatorname{ann}_{X}(A)$. On the other hand, $0=\alpha(b)=w^{\prime} b(b \in B)$ so that $w^{\prime} \in a n n_{X}(B)$. Thus

$$
w=\left(w-w^{\prime}\right)+w^{\prime} \in a n n_{X}(A)+a n n_{X}(B) .
$$

It follows that $a n n_{X}(A \cap B) \subseteq a n n_{X}(A)+a n n_{X}(B)$ and hence $a n n_{X}(A \cap B)=a n n_{X}(A)+a n n_{X}(B)$.

Note that if $R$ is a ring and $A$ and $B$ are right ideals of $R$ such that a right $R$-module $X$ is $\{A+B\}-R_{R^{-}}$ injective then it does not follow that $X$ is $\{A\}-R_{R}$-injective. For example, let $X$ be any $R$-module which is not injective. Then there exists a right ideal $A$ of $R$ and a homomorphism $\varphi: A \rightarrow X$ which does not lift to $R$. In other words, $X$ is not $\{A\}-R_{R}$-injective. If $B=R$ then $A+B=R$ and $X$ is clearly $\{A+B\}-R_{R}$-injective. 


\section{$2 n$-Injective modules}

Let $R$ be any ring and let $n$ be any positive integer. Let $\mathfrak{F}_{n}$ denote the collection of $n$-generated right ideals of $R$ and let $\mathfrak{F}$ denote the collection of finitely generated right ideals of $R$. Note that $\mathfrak{F}_{n} \subseteq \mathfrak{F}_{n+1}$ for every positive integer $n$ and that $\mathfrak{F}=\cup_{n \geq 1} \mathfrak{F}_{n}$. Following [9, p. 103], we shall call a (right) $R$-module $X n$-injective provided it is $\mathfrak{F}_{n}-R_{R}$-injective. Note that in [9], 1 -injective modules are also called $P$-injective. Clearly $(n+1)$-injective modules are $n$-injective for every positive integer $n$. However, it is pointed out in [9, Example 5.22] that an example of Björk [1] (see also [14]) is 1-injective but not 2-injective. We shall give more details of Björk's example at the end of the paper. An $R$-module $X$ will be called $f$-injective in case it is $\mathfrak{F}-R_{R}$-injective. Thus a module is $f$-injective if and only if it is $n$-injective for every positive integer $n$.

It is easy to prove that a ring $R$ is von Neumann regular if and only if every right (or left) $R$-module is 1 -injective, and in this case every right (or left) $R$-module is $f$-injective. Clearly every direct sum of $f$-injective modules is $f$-injective. Therefore, by the Bass-Papp Theorem (see, for example, [16, Theorem 4.1]), a ring $R$ is right Noetherian if and only if every $f$-injective right $R$-module is injective. A ring $R$ is called right semihereditary provided every finitely generated right ideal is projective. Commutative semihereditary domains are called Prüfer domains. There is an extensive literature on Prüfer domains. Note that Corollary 1.9 with $\mathfrak{A}=\mathfrak{F}$ gives at once: a ring $R$ is right semihereditary if and only if every homomorphic image of an $f$-injective right $R$-module is $f$-injective.

Let $n$ be any positive integer and let $a_{i}(1 \leq i \leq n)$ be any collection of $n$ (not necessarily distinct) elements of $R$. Let $X$ be an $R$-module. Then we shall denote by $\mathcal{C}_{X}\left(a_{1}, \ldots, a_{n}\right)$ the set of elements $\left(x_{1}, \ldots, x_{n}\right)$ of the direct sum $X^{n}$ of $n$ copies of $X$ with the property that

$$
x_{1} r_{1}+\cdots+x_{n} r_{n}=0,
$$

for all elements $r_{i} \in R(1 \leq i \leq n)$ such that

$$
a_{1} r_{1}+\cdots+a_{n} r_{n}=0 .
$$

In addition, we shall denote by $X\left(a_{1}, \ldots, a_{n}\right)$ the set of elements $\left(x a_{1}, \ldots, x a_{n}\right)$ of $X^{n}$, where $x$ runs through the elements of $X$. Clearly,

$$
X\left(a_{1}, \ldots, a_{n}\right) \subseteq \mathcal{C}_{X}\left(a_{1}, \ldots, a_{n}\right) .
$$

Theorem 2.1 Let $R$ be a ring, let $a_{i}(1 \leq i \leq n)$ be elements of $R$, for some positive integer $n$ and let $A$ be the right ideal $a_{1} R+\cdots+a_{n} R$ of $R$. Then an $R$-module $X$ is $\{A\}-R_{R}$-injective if and only if $X\left(a_{1}, \ldots, a_{n}\right)=\mathcal{C}_{X}\left(a_{1}, \ldots, a_{n}\right)$.

Proof Suppose first that $X$ is $\{A\}-R_{R}$-injective. Let $\left(x_{1}, \ldots, x_{n}\right) \in \mathcal{C}_{X}\left(a_{1}, \ldots, a_{n}\right)$, for some $x_{i} \in X(1 \leq$ $i \leq n)$. Define a mapping $\varphi: A \rightarrow X$ by

$$
\varphi\left(a_{1} r_{1}+\cdots+a_{n} r_{n}\right)=x_{1} r_{1}+\cdots+x_{n} r_{n},
$$

for any elements $r_{i} \in R(1 \leq i \leq n)$. Because $\left(x_{1}, \ldots, x_{n}\right) \in \mathcal{C}_{X}\left(a_{1}, \ldots, a_{n}\right), \varphi$ is well-defined and in this case $\varphi$ is clearly a homomorphism. By hypothesis, $\varphi$ lifts to a homomorphism $\theta: R \rightarrow X$. Let $x=\theta(1)$. Then $x_{i}=\varphi\left(a_{i}\right)=\theta\left(a_{i}\right)=\theta(1) a_{i}=x a_{i}$ for all $1 \leq i \leq n$ and hence

$$
\left(x_{1}, \ldots, x_{n}\right)=\left(x a_{1}, \ldots, x a_{n}\right) \in X\left(a_{1}, \ldots, a_{n}\right) .
$$

Thus $\mathcal{C}_{X}\left(a_{1}, \ldots, a_{n}\right) \subseteq X\left(a_{1}, \ldots, a_{n}\right)$ and hence $\mathcal{C}_{X}\left(a_{1}, \ldots, a_{n}\right)=X\left(a_{1}, \ldots, a_{n}\right)$.

Conversely, suppose that $\mathcal{C}_{X}\left(a_{1}, \ldots, a_{n}\right)=X\left(a_{1}, \ldots, a_{n}\right)$. Let $\alpha: A \rightarrow X$ be any homomorphism. Let $y_{i}=\alpha\left(a_{i}\right) \in X(1 \leq i \leq n)$. If $s_{i} \in R(1 \leq i \leq n)$ and $a_{1} s_{1}+\cdots+a_{n} s_{n}=0$ then

$$
y_{1} s_{1}+\cdots+y_{n} s_{n}=\alpha\left(a_{1}\right) s_{1}+\cdots+\alpha\left(a_{n}\right) s_{n}=\alpha\left(a_{1} s_{1}+\cdots+a_{n} s_{n}\right)=\alpha(0)=0 .
$$

Thus $\left(y_{1}, \ldots, y_{n}\right) \in \mathcal{C}_{X}\left(a_{1}, \ldots, a_{n}\right)=X\left(a_{1}, \ldots, a_{n}\right)$ and there exists $y \in X$ with $y_{i}=y a_{i}(1 \leq i \leq n)$. It follows that the homomorphism $\beta: R \rightarrow X$ defined by $\beta(t)=y t(t \in R)$ lifts $\alpha$ to $R$. Thus $X$ is $\{A\}-R_{R}$-injective.

Corollary 2.2 Given a ring $R$ and a positive integer $n$, an $R$-module $X$ is $n$-injective if and only if $X\left(a_{1}, \ldots, a_{n}\right)=\mathcal{C}_{X}\left(a_{1}, \ldots, a_{n}\right)$ for all elements $a_{i}(1 \leq i \leq n)$ of $R$. 
Proof By Theorem 2.1.

Given a non-empty subset $T$ of a ring $R, \mathbf{r}(T)$ will denote the set of elements $r \in R$ such that $t r=0$ for all $t \in T$. In case $T=\{t\}$, for some element $t \in R$, we write $\mathbf{r}(T)$ simply as $\mathbf{r}(t)$. Note that $\mathbf{r}(T)$ is a right ideal of $R$ for every non-empty subset $T$ of $R$. If $a$ is an element of $R$ and $X$ an $R$-module then we shall denote by $X a$ the set of elements $x a(x \in X)$ of $X$. Theorem 2.1 has the following further consequence:

Corollary 2.3 Given an element a of a ring $R$, an $R$-module $X$ is $\{a R\}-R_{R}$-injective if and only if $X a=$ $a n n_{X}(\boldsymbol{r}(a))$. Moreover, $X$ is 1-injective if and only if $X b=a n_{X}(\boldsymbol{r}(b))$ for all $b \in R$.

Proof By Theorem 2.1.

The next result gives another characterization of $n$-injective modules, for any integer $n \geq 2$.

Theorem 2.4 Let $R$ be any ring and let $n \geq 2$ be a positive integer. Then the following statements are equivalent for an $R$-module $X$ :

(i) $X$ is n-injective.

(ii) $X$ is 1-injective and $a n n_{X}(A \cap B)=a n n_{X}(A)+a n n_{X}(B)$ for all right ideals $A$ and $B$ of $R$ such that the right ideals $A, B$ and $A+B$ are all n-generated.

(iii) $X$ is 1 -injective and for all elements $a_{i} \in R(1 \leq i \leq n)$,

$$
\operatorname{ann}_{X}\left(\left(a_{1} R+\cdots+a_{n-1} R\right) \cap a_{n} R\right)=\operatorname{ann}_{X}\left(a_{1} R+\cdots+a_{n-1} R\right)+\operatorname{ann}_{X}\left(a_{n} R\right) .
$$

Proof (i) $\Rightarrow$ (ii) Clearly $X$ is 1-injective. Moreover, if $A$ and $B$ are right ideals of $R$ such that $A, B$ and $A+B$ are all $n$-generated then $X$ is $\{A\}-R_{R}$-injective, $X$ is $\{B\}-R_{R}$-injective and $X$ is $\{A+B\}-R_{R}$-injective. By Theorem 1.10, $a n n_{X}(A \cap B)=a n n_{X}(A)+a n n_{X}(B)$.

(ii) $\Rightarrow$ (iii) Clear.

(iii) $\Rightarrow$ (i) We prove that $X$ is $n$-injective by induction on $n$. Let $C$ be any 2-generated right ideal of $R$. Then $C=c_{1} R+c_{2} R$ for some elements $c_{1}, c_{2} \in C$. By hypothesis, $a n n_{X}\left(c_{1} R \cap c_{2} R\right)=a n n_{X}\left(c_{1} R\right)+a n n_{X}\left(c_{2} R\right)$, so that $X$ is $\{C\}-R_{R}$-injective by Theorem 1.10. It follows that $X$ is 2-injective. Now suppose that $n \geq 3$. By induction on $n$ we can suppose that $X$ is $(n-1)$-injective. Let $D$ be any $n$-generated right ideal of $R$. Then there exist an (n-1)-generated right ideal $E$ and a principal right ideal $F$ such that $D=E+F$. Now $X$ is both $\{E\}-R_{R}$-injective and $\{F\}-R_{R}$-injective and by hypothesis $\operatorname{ann}_{X}(E \cap F)=\operatorname{ann}_{X}(E)+\operatorname{ann}_{X}(F)$. By Theorem 1.10, $X$ is $\{D\}-R_{R}$-injective. It follows that $X$ is $n$-injective.

Compare the final result of this section with the Ikeda-Nakayama Lemma (see, for example, [9, Lemma $1.37])$.

Corollary 2.5 Let $R$ be any ring. An $R$-module $X$ is $f$-injective if and only if $X$ is 1-injective and ann $n_{X}(A \cap$ $B)=\operatorname{ann}_{X}(A)+a n n_{X}(B)$ for all finitely generated right ideals $A$ and $B$ of $R$.

Proof By Theorem 2.4.

\section{Divisible modules}

Given a non-empty subset $S$ of an $R$-module $M, \operatorname{ann}_{R}(S)$ will denote the set of elements $r \in R$ such that $s r=0$ for all $s \in S$. Note that $a_{n n}(S)$ is a right ideal of $R$. In case $S=\{m\}$, for some element $m$ of $M, a n n_{R}(S)$ will be written simply as $a n n_{R}(m)$.

If $R$ is a commutative domain then an $R$-module $M$ is called divisible provided $M=M c$ for every non-zero element $c \in R$. There is an extensive theory of divisible modules over commutative domains stretching back to [7]. More generally, in [16, p. 32] or [19, p. 132] given a (not necessarily commutative) ring $R$, a right $R$-module $M$ is called divisible provided $M=M c$ for every element $c \in R$ with $\mathbf{r}(c)=0$. Sharpe and Vamos [16, Lemmas 2.4 and 2.5 and Proposition 2.6] note that every homomorphic image of a divisible module is divisible, every direct product and every direct sum of divisible modules is divisible and every injective module is divisible. Moreover, in case $R$ is a commutative domain then every torsion-free divisible $R$-module is injective (see [16, Proposition 2.7]). A commutative domain $R$ is Dedekind if and only if every divisible $R$-module is injective (see [16, Theorem 4.25]). Note the following simple fact: 
Lemma 3.1 Let $R$ be any ring and let $\Re$ denote the collection of right ideals of $R$ of the form $c R$ where $c \in R$ and $\boldsymbol{r}(c)=0$. Then a right $R$-module $X$ is divisible if and only if $X$ is $\Re-R_{R}$-injective.

Proof Suppose first that $X$ is divisible. Let $c$ be any element of $R$ such that $\mathbf{r}(c)=0$. Let $\varphi: c R \rightarrow X$ be any homomorphism. Then $\varphi(c)=x c$ for some element $x \in X$. It follows that $\varphi$ lifts to $R$. Conversely, suppose that $X$ is $\Re-R_{R}$-injective. Let $u \in X$ and $d \in R$ with $\mathbf{r}(d)=0$. Define a mapping $\theta: d R \rightarrow X$ by $\theta(d s)=u s$ for all $s \in R$. It is easy to check that $\theta$ is well defined and a homomorphism. By hypothesis, $\theta$ lifts to a homomorphism $\sigma: R \rightarrow X$. Let $v=\sigma(1) \in X$. Then

$$
u=\theta(d)=\sigma(d)=\sigma(1) d=v d .
$$

It follows that $X=X d$ and that $X$ is divisible.

Let $R$ be any ring. There are various other notions of 'divisible module' in the literature. Levy [4] calls an $R$-module $M$ divisible provided $M=M c$ for every element $c$ in $R$ such that $\mathbf{r}(c)=0$ and $\mathbf{l}(c)=0$ where $\mathbf{I}(c)=\{r \in R: r c=0\}$. In [5], an $R$-module $X$ is called divisible provided it is 1 -injective. Given an hereditary torsion theory $\tau$ on the category Mod- $R$ of right $R$-modules, a right $R$-module $X$ is called $\tau$-divisible in [8] (or [10] or [17]) provided, for every right $R$-module $M, X$ is $\mathfrak{D}-M$-injective. Here $\mathfrak{D}$ is the collection of $\tau$-dense submodules of $M$, where, as usual, a submodule $L$ of $M$ is $\tau$-dense when $M / L$ is a $\tau$-torsion module. However, in [18], $\tau$-divisible modules are somewhat different.

Let $R$ be a ring and let $M$ be an $R$-module. Let $n$ be a positive integer and let $a_{i} \in R(1 \leq i \leq n)$. Then we define

$$
M a_{1}+\cdots+M a_{n}=\left\{m_{1} a_{1}+\cdots+m_{n} a_{n}: m_{i} \in M(1 \leq i \leq n)\right\} .
$$

Note that $M a_{1}+\cdots+M a_{n}$ is, in general, merely a subgroup of the Abelian group $(M,+)$. It is clear that

$$
M a_{1}+\cdots+M a_{n} \subseteq \operatorname{ann}_{M}\left(\mathbf{r}\left(R a_{1}+\cdots+R a_{n}\right)\right) .
$$

We shall call the module Mn-divisible in case

$$
M a_{1}+\cdots+M a_{n}=\operatorname{ann}_{M}\left(\mathbf{r}\left(R a_{1}+\cdots+R a_{n}\right)\right)
$$

for all elements $a_{i} \in R(1 \leq i \leq n)$. Note that the module $M$ is $n$-divisible if and only if, given $m \in M, m \in$ $M a_{1}+\cdots+M a_{n}$ for all elements $a_{i} \in R(1 \leq i \leq n)$ with $\mathbf{r}\left(R a_{1}+\cdots+R a_{n}\right) \subseteq \operatorname{ann}_{R}(m)$. We shall call the module $M f$-divisible provided $M$ is $n$-divisible for every positive integer $n$. It is clear that every $(n+1)$-divisible module is $n$-divisible for every positive integer $n$.

In particular, an $R$-module $X$ is 1-divisible if and only if $X c=a n_{X}(\mathbf{r}(c))$ for all $c \in R$. Note that Facchini [3, p. 387] calls a 1-divisible module (over a commutative ring) simply "divisible" (see also [12, p. 1225]). This gives immediately by Corollary 2.3:

Lemma 3.2 Let $R$ be any ring. Then an $R$-module $X$ is 1-divisible if and only if $X$ is 1-injective.

Note that every 1-divisible module is divisible in the sense of [16]. The converse is false, as the following example shows:

Example 3.3 Let $R$ be any commutative ring which contains a (non-zero) maximal ideal $A$ with $A$ not a principal ideal and $A^{2}=0$. Then every $R$-module is divisible but the $R$-module $R$ is not 1-divisible.

Proof Let $c \in R$ with $\mathbf{r}(c)=0$. Then $c \notin A$ and hence $c$ is a unit in $R$. It follows that every $R$-module is divisible. However, let $0 \neq a \in A$. Then $\mathbf{r}(a)=A$ and hence $a n n_{R}(\mathbf{r}(a))=A \neq R a$. Thus the $R$-module $R$ is not 1-divisible.

It is easy to give an example of a ring $R$ satisfying the properties of Example 3.3. We offer one such. Let $F$ be any field and let $V$ be any vector space over $F$ of dimension at least 2. Let $R$ denote the trivial extension of $V$ by $F$. Thus the elements of $R$ are ordered pairs $(f, v)$ with $f \in F$ and $v \in V$. Addition and multiplication are given by

$$
\left(f_{1}, v_{1}\right)+\left(f_{2}, v_{2}\right)=\left(f_{1}+f_{2}, v_{1}+v_{2}\right), \quad \text { and }\left(f_{1}, v_{1}\right)\left(f_{2}, v_{2}\right)=\left(f_{1} f_{2}, f_{2} v_{1}+f_{1} v_{2}\right),
$$

for all $f_{1}, f_{2} \in F$ and $v_{1}, v_{2} \in V$. It is easy to check that $R$ has the required properties with $A$ the set of elements of $R$ of the form $(0, v)(v \in V)$. 
Theorem 3.4 Let $R$ be a ring, let $n$ be a positive integer, let $F$ denote the free $R$-module $R_{R}^{n}$ and let $\mathfrak{C}$ denote the collection of cyclic submodules of $F$. Then an $R$-module $M$ is $n$-divisible if and only if $M$ is $\mathfrak{C}-F$-injective.

Proof Suppose first that $M$ is $n$-divisible. Let $f \in F$. Then $f=\left(a_{1}, \ldots, a_{n}\right)$ for some $a_{i} \in R(1 \leq i \leq n)$. Let $\varphi: f R \rightarrow M$ be any homomorphism. Let $m=\varphi(f) \in M$. Then $r \in \mathbf{r}\left(R a_{1}+\cdots+R a_{n}\right)$ implies that $f r=0$ and hence $m r=\varphi(f) r=\varphi(f r)=0$. Thus $m \in \operatorname{ann}_{M}\left(\mathbf{r}\left(R a_{1}+\cdots+R a_{n}\right)\right)=M a_{1}+\cdots+M a_{n}$, by hypothesis. There exist elements $m_{i} \in M(1 \leq i \leq n)$ such that $m=m_{1} a_{1}+\cdots+m_{n} a_{n}$. Define a mapping $\theta: F \rightarrow M$ by

$$
\theta\left(r_{1}, \ldots, r_{n}\right)=m_{1} r_{1}+\cdots+m_{n} r_{n},
$$

for all $r_{i} \in R(1 \leq i \leq n)$. Then $\theta$ is clearly a homomorphism. Moreover, for all $s \in R$ we have that

$$
\theta(f s)=m_{1} a_{1} s+\cdots+m_{n} a_{n} s=m s=\varphi(f s) .
$$

Thus $\theta$ lifts $\varphi$ to $F$.

Conversely, suppose that $M$ is $\mathfrak{C}-F$-injective. Let $c_{i} \in R(1 \leq i \leq n)$ and let $u \in \operatorname{ann}_{M}\left(\mathbf{r}\left(R c_{1}+\cdots+\right.\right.$ $\left.\left.R c_{n}\right)\right)$. Let $g=\left(c_{1}, \ldots, c_{n}\right) \in F$. Define a mapping $\alpha: g R \rightarrow M$ by $\alpha(g t)=u t(t \in R)$. Note that if $g t=0$ then $t \in \mathbf{r}\left(R c_{1}+\cdots+R c_{n}\right)$ and hence $u t=0$. Thus the mapping $\alpha$ is well-defined and it is not difficult to see that $\alpha$ is a homomorphism. By hypothesis $\alpha$ can be lifted to a homomorphism $\beta: F \rightarrow M$. For each integer $1 \leq i \leq n$, let $f_{i}$ denote the element of $F$ whose $i$ th component is 1 and whose other components are all 0 . Then

$$
u=\alpha(g)=\beta(g)=\beta\left(c_{1}, \ldots, c_{n}\right)=\beta\left(f_{1} c_{1}+\cdots+f_{n} c_{n}\right),
$$

and hence

$$
u=\beta\left(f_{1}\right) c_{1}+\cdots+\beta\left(f_{n}\right) c_{n} \in M c_{1}+\cdots+M c_{n} .
$$

It follows that $\operatorname{ann}_{M}\left(\mathbf{r}\left(R c_{1}+\cdots+R c_{n}\right)\right) \subseteq M c_{1}+\cdots+M c_{n}$ and hence $\operatorname{ann}_{M}\left(\mathbf{r}\left(R c_{1}+\cdots+R c_{n}\right)\right)=$ $M c_{1}+\cdots+M c_{n}$. Thus $M$ is $n$-divisible.

Note the following result to be found in [11, Lemma 2.3] which is a generalization of [16, Proposition 2.6].

Corollary 3.5 Let $R$ be any ring. Then every injective $R$-module is $f$-divisible.

Proof By Theorem 3.4.

Combining Theorem 3.4 with the results in Sect. 1 we can immediately obtain a number of further corollaries.

Corollary 3.6 Let $R$ be a ring and $n$ a positive integer. Then every direct product and every direct sum of $n$-divisible $R$-modules is n-divisible.

Proof By Lemma 1.2, Corollary 1.3 and Theorem 3.4.

Corollary 3.7 Let $R$ be a ring and $n$ a positive integer. Let $Y$ be a submodule of an $R$-module $X$ such that $Y$ and $X / Y$ are both $n$-divisible. Then $X$ is $n$-divisible.

Proof By Lemma 1.5 and Theorem 3.4.

For any ring $R$ and free $R$-module $F$, we let $\mathfrak{C}_{F}$ denote the collection of cyclic submodules of $F$.

Corollary 3.8 Let $R$ be a ring and let $G$ be the free $R$-module $R_{R}^{\aleph_{0}}$. Then the following statements are equivalent for an $R$-module $X$.

(i) $X$ is $f$-divisible.

(ii) $X$ is $\mathfrak{C}_{F}-F$-injective for every non-finitely generated free $R$-module $F$.

(iii) $X$ is $\mathfrak{C}_{G}-G$-injective. 
Proof (i) $\Rightarrow$ (ii) Let $f \in F$. Then there exist a direct summand $F^{\prime}$ of $F$ and a positive integer $n$ such that $F^{\prime} \cong R_{R}^{n}$ and $f \in F^{\prime}$. Let $\varphi: f R \rightarrow X$ be any homomorphism. Because $X$ is $n$-divisible, Theorem 3.4 gives that $\varphi$ lifts to $F^{\prime}$ and hence also to $F$. It follows that $X$ is $\mathfrak{C}_{F}-F$-injective.

(ii) $\Rightarrow$ (iii) Clear.

(iii) $\Rightarrow$ (i) Suppose that $X$ is $\mathfrak{C}_{G}-G$-injective. For any positive integer $m, X$ is $\mathfrak{C}_{m}-R_{R}^{m}$-injective, where $\mathfrak{C}_{m}$ denotes the collection of cyclic submodules of $R_{R}^{m}$. By Theorem 3.4, $X$ is $m$-divisible for every positive integer $m$ and hence $X$ is $f$-divisible.

There are analogues of Corollaries 3.6 and 3.7 for $f$-divisible $R$-modules. A ring $R$ is called a right $P P$-ring provided every principal right ideal is projective. For example (not necessarily commutative) domains are right and left PP-rings. Recall the following well-known lemma which is included for completeness:

Lemma 3.9 Let $R$ be a right PP-ring. Then every cyclic submodule of every free $R$-module is projective.

Proof It is sufficient to prove the result for free $R$-modules of finite rank. Let $F$ be a free $R$-module of rank $n \geq 1$ and let $C$ be a cyclic submodule of $F$. If $n=1$ then $F \cong R_{R}$ and hence $C$ is projective. Suppose that $n \geq 2$. There exists a free submodule $G$ of $F$ of rank $n-1$ and a submodule $L$ of $F$ such that $L \cong R_{R}$ and $F=G \oplus L$. Let $\pi: F \rightarrow L$ denote the canonical projection. Then $\pi(C)$ is a cyclic submodule of $L$ so that $\pi(C)$ is projective. It follows that $C \cong(C \cap G) \oplus \pi(C)$. Because $C \cap G$ is a homomorphic image of $C$, we have that $C \cap G$ is a cyclic submodule of the free module $G$. By induction on $n, C \cap G$ is a projective module and hence so too is $C$.

In [6] it is proved that the following statements are equivalent:

(i) $R$ is a right PP-ring.

(ii) Every homomorphic image of a 1-injective right $R$-module is 1-injective.

(iii) Every homomorphic image of an injective right $R$-module is 1 -injective.

Compare the following result.

Theorem 3.10 The following statements are equivalent for a ring $R$.

(i) $R$ is a right PP-ring.

(ii) Every homomorphic image of a 1-divisible right $R$-module is 1-divisible.

(iii) For every positive integer $n$, every homomorphic image of an $n$-divisible right $R$-module is $n$-divisible.

(iv) Every homomorphic image of an $f$-divisible right $R$-module is $f$-divisible.

(v) Every homomorphic image of an injective right $R$-module is $f$-divisible.

Proof (i) $\Leftrightarrow$ (ii) By Corollary 1.8, (i) holds if and only if every homomorphic image of a $\mathfrak{C}-R_{R}$-injective module is $\mathfrak{C}-R_{R}$-injective, where $\mathfrak{C}$ denotes the collection of cyclic submodules of $R_{R}$. Now apply Theorem 3.4 .

(i) $\Rightarrow$ (iii) Let $n$ be any positive integer. Because $R$ is a right PP-ring, Lemma 3.9 gives that every cyclic submodule of $R_{R}^{n}$ is projective. By Corollary 1.8 and Theorem 3.4, it follows that every homomorphic image of an $n$-divisible $R$-module is $n$-divisible.

(iii) $\Rightarrow$ (iv) Clear.

(iv) $\Rightarrow$ (v) By Corollary 3.5 .

(v) $\Rightarrow$ (i) By Corollaries 1.8 and 3.8.

Note that the ring $R$ in Example 3.3 has the property that every homomorphic image of every divisible module is divisible but $R$ is not a PP-ring because $a R$ is not a projective module for each $0 \neq a \in A$. If $R$ is a (not necessarily commutative) domain then it is clear that the following statements are equivalent for an $R$-module $X$ :

(i) $X$ is divisible.

(ii) $X$ is 1-divisible.

(iii) $X$ is $f$-divisible.

Now we show more generally that if $R$ is a right PP-ring then every 1-divisible $R$-module is $f$-divisible.

Theorem 3.11 Let $R$ be a right PP-ring. Then every 1-divisible right $R$-module is $f$-divisible. 
Proof Let $X$ be a 1-divisible $R$-module. We shall show that $X$ is $n$-divisible, for every positive integer $n$, by induction on $n$. Note that it is given that $X$ is 1-divisible. Now suppose that $X$ is $n$-divisible for some positive integer $n$. Let $F$ denote the free $R$-module $R_{R}^{(n+1)}$. Let $a_{i} \in R(1 \leq i \leq n+1)$, let $a=\left(a_{1}, \ldots, a_{n+1}\right) \in F$ and let $\varphi: a R \rightarrow X$ be any homomorphism. Because $a_{1} R$ is a projective $R$-module, there exists an idempotent $e \in R$ such that $\mathbf{r}\left(a_{1}\right)=e R$. Then it can easily be checked that $a R=b R \oplus c R$, where $b=a(1-e)=$ $\left(a_{1}, a_{2}(1-e), \ldots, a_{n+1}(1-e)\right)$ and $c=a e=\left(0, a_{2} e, \ldots, a_{n+1} e\right)$. By hypothesis, $X$ is $n$-divisible and hence the homomorphism $\left.\varphi\right|_{c R}: c R \rightarrow X$ lifts to a homomorphism $\alpha: H \rightarrow X$ by Theorem 3.4, where $H$ is the submodule $0 \oplus R_{R}^{n}$ of $F$. Note that $b R \cap H=0$. It follows that the mapping $\beta: b R \oplus H \rightarrow X$ defined by $\beta(b r+h)=\varphi(b r)+\alpha(h)$ for all $r \in R$ and $h \in H$ is a homomorphism such that $\beta(a s)=\varphi(a s)$ for all $s \in R$.

Next note that $b R \oplus H=d R \oplus H$ where $d=\left(a_{1}, 0,0, \ldots, 0\right) \in F$. Because $X$ is 1-injective, the homomorphism $\left.\beta\right|_{d R}: d R \rightarrow X$ lifts to a homomorphism $\gamma: G \rightarrow X$, where $G$ is the submodule $R \oplus 0^{n}$ of $F$. But $F=G \oplus H$ and the homomorphism $\theta: F \rightarrow X$ defined by $\theta(g+h)=\gamma(g)+\alpha(h)$, for all $g \in G, h \in H$, lifts $\beta$, and hence also $\varphi$, to $F$. By Theorem 3.4, $X$ is $(n+1)$-divisible. Finally, by induction, $X$ is $f$-divisible, as required.

Next we shall give another situation in which a 1-divisible module is $n$-divisible, for some positive integer $n \geq 2$.

Proposition 3.12 Let $R$ be any ring, let $n$ be a positive integer and let $X$ be a 1 -divisible $R$-module such that $X$ is $\boldsymbol{r}\left(R a_{1}+\cdots+R a_{n}\right)-R_{R}$-injective for all elements $a_{i} \in R(1 \leq i \leq n)$. Then $X$ is $n$-divisible.

Proof We prove the result by induction on $n$. It is given that $X$ is 1-divisible. Suppose that $n \geq 2$ and the result holds for $n-1$. Clearly the module $X$ is $\mathbf{r}\left(R a_{1}+\cdots+R a_{n-1}\right)-R_{R}$-injective for all elements $a_{i} \in R(1 \leq$ $i \leq n-1)$. By induction, $X$ is $(n-1)$-divisible. Let $b_{i} \in R(1 \leq i \leq n)$ and let $B=R b_{1}+\cdots+R b_{n-1}$. Then

$$
X b_{1}+\cdots+X b_{n-1}=a n n_{X}\left(\mathbf{r}\left(R b_{1}+\cdots+R b_{n-1}\right)\right)=a n n_{X}(\mathbf{r}(B)),
$$

and hence

$$
X b_{1}+\cdots+X b_{n-1}+X b_{n}=a n n_{X}(\mathbf{r}(B))+a n n_{X}\left(\mathbf{r}\left(R b_{n}\right)\right) .
$$

By the proof of Theorem 1.10,

$$
a n n_{X}(\mathbf{r}(B))+a n n_{X}\left(\mathbf{r}\left(R b_{n}\right)=a n n_{X}\left(\mathbf{r}(B) \cap \mathbf{r}\left(R b_{n}\right)\right)\right.
$$

and hence

$$
X b_{1}+\cdots+X b_{n}=a n n_{X}\left(\mathbf{r}\left(B+R b_{n}\right)\right)=a n_{X}\left(\mathbf{r}\left(R b_{1}+\cdots+R b_{n}\right)\right) .
$$

It follows that $X$ is $n$-divisible.

For any non-empty subset $T$ of a ring $R$ we denote by $\mathbf{l}(T)$ the set of elements $r \in R$ such that $r t=0$ for all $t \in T$. Note that $\mathbf{I}(T)$ is a left ideal of $R$. A left ideal $L$ of $R$ is called a left annihilator provided $L=\mathbf{I}(T)$ for some non-empty subset $T$ of $R$. Note the following simple fact:

Proposition 3.13 Given a ring $R$ and a positive integer $n$, the module $R_{R}$ is $n$-divisible if and only if every $n$-generator left ideal of $R$ is a left annihilator.

Proof The module $R_{R}$ is $n$-divisible if and only if for all $a_{i} \in R(1 \leq i \leq n)$,

$$
R a_{1}+\cdots+R a_{n}=\operatorname{ann}_{R}\left(\mathbf{r}\left(R a_{1}+\cdots+R a_{n}\right)\right)=\mathbf{l}\left(\mathbf{r}\left(R a_{1}+\cdots+R a_{n}\right)\right),
$$

and the result follows by well-known standard arguments.

Finally we give more details of Björk's example mentioned above. Let $F$ be any field such that there exists a monomorphism $\alpha: F \rightarrow F$ such that $\alpha(F) \neq F$. Let $R$ denote the ring whose elements are the ordered pairs $(a, b)$, with $a, b \in F$, with addition and multiplication being defined by

$$
(a, b)+(c, d)=(a+c, b+d) \text { and }(a, b)(c, d)=(a c, a d+b \alpha(c))
$$


for all $a, b, c, d \in F$. It is easy to check that $R$ is a ring with identity $(1,0)$ and with Jacobson radical $J=\{(0, b): b \in F\}$. Moreover, $R / J \cong F, J^{2}=0$ and $J=R(0,1)$ is a minimal left ideal of $R$. Thus the left ideals of $R$ form a composition series $R \supset J \supset 0$. It is not difficult to check that the right $R$-module $R$ is 1-injective but the left $R$-module $R$ is not (see [9, Example 5.22] for more details). Moreover, if $f, g \in F$ such that $\alpha(F) f \cap \alpha(F) g=0$ then $(0, f) R \cap(0, g) R=0$ in $R$ and if $\pi:(0, f) R \oplus(0, g) R \rightarrow R$ is the homomorphism given by projection onto the first component then $\pi$ does not lift to $R$. Thus $R_{R}$ is not 2-injective. Not also that this shows that $\mathfrak{A}_{R}\left(R_{R}\right)$ is not closed under taking direct sums. Now we prove

Corollary 3.14 With the above notation, $R$ is not a right PP-ring, the right $R$-module $R$ is $f$-divisible but not 2-injective. Moreover the left $R$-module $R$ is not 1-divisible.

Proof We have already remarked that $R_{R}$ is not 2-injective. The only left ideals of $R$ are $0, J$ and $R$, each of which is clearly a left annihilator. By Proposition $3.13, R_{R}$ is $f$-divisible. Now consider the element $(0,1)$ of $R$. Note that $(0,1) R=\{(0, h): h \in \alpha(F)\}$. However, $\mathbf{l}((0,1) R)=J$ and hence $\mathbf{r}(\mathbf{l}((0,1) R)=J \neq(0,1) R$. Again using Proposition 3.13, we see that ${ }_{R} R$ is not 1 -divisible. It is easy to check that the only idempotents in $R$ are $(0,0)$ and $(1,0)$ and $\mathbf{r}(0,1)=\{(0, f): f \in F\}$ so that $(0,1) \mathrm{R}$ is not projective. Thus $R$ is not a right PP-ring.

We do not know an example of a 1-divisible module which is not 2-divisible.

Open Access This article is distributed under the terms of the Creative Commons Attribution License which permits any use, distribution, and reproduction in any medium, provided the original author(s) and the source are credited.

\section{References}

1. Björk, J.-E.: Rings satisfying certain chain conditions. J. Reine Angew. Math. 245, 63-73 (1970)

2. Bunu, I.D.: On the concept of injectivity in a category of modules (Russian). Rings and modules. Mat. Issled. Vyp. 38, 71-85, 205-206 (1976)

3. Facchini, A.: Relative injectivity and pure-injective modules over Prüfer rings. J. Algebra 110(2), 380-406 (1987)

4. Levy, L.: Torsion-free and divisible modules over non-integral-domains. Can. J. Math. 15, 132-151 (1963)

5. Mao, L.; Ding, N.: On divisible and torsion-free modules. Commun. Algebra 36(2), 708-731 (2008)

6. Mao, L.; Ding, N.; Tong, W.: New characterizations and generalizations of PP rings. Vietnam J. Math. 33(1), 97-110 (2005)

7. Matlis, E.: Divisible modules. Proc. Am. Math. Soc. 11, 385-391 (1960)

8. Morimoto, S.: Weakly divisible and divisible modules. Tsukuba J. Math. 6(2), 195-200 (1982)

9. Nicholson, W.K.; Yousif, M.F.: Quasi-Frobenius Rings. Cambridge Tracts in Mathematics, vol. 158. Cambridge University Press, Cambridge (2003)

10. Nishida, K.: Divisible modules, codivisible modules and quasi-divisible modules. Commun. Algebra 5(6), 591-610 (1977)

11. Özcan, A.C.; Smith, P.F.: The $Z^{*}$ functor for rings whose primitive images are Artinian. Commun. Algebra 30(10), 49154930 (2002)

12. Puninski, G.: Pure-injective and RD-injective envelopes of a ring. Math. Notes 52(6), 1224-1230 (1992)

13. Ramamurthi, V.S.; Rangaswamy, K.M.: On finitely injective modules. Collection of articles dedicated to the memory of Hanna Neumann II. J. Austral. Math. Soc. 16, 239-248 (1973)

14. Rutter, E.A.: Rings with the principal extension property. Commun. Algebra 3, 203-212 (1975)

15. Salce, L.: On finitely injective modules and locally pure-injective modules over Prüfer domains. Proc. Am. Math. Soc. 135(11), 3485-3493 (2007)

16. Sharpe D.W.; Vamos P.: Injective Modules. Cambridge Tracts in Mathematics, vol. 62. Cambridge University Press, Cambridge (1972)

17. Takehana, Y.: A preradical which satisfies the property that every weakly divisible module is divisible. Tsukuba J. Math. 5(1), 153-163 (1981)

18. Teply, M.L.: A class of divisible modules. Pac. J. Math. 45(2), 653-668 (1973)

19. Wisbauer, R.: Foundations of Module and Ring Theory. Gordon and Breach, Philadelphia (1991) 